\title{
Sinergie Italian Journal of Management, formerly Sinergie Rivista di Studi e Ricerche
}

Giovanni Panati, fondatore della rivista, amava sottolineare come l'unica costante dell'ambiente fosse il cambiamento, a volte mosso nella direzione di una meta definita, a volte vagante senza meta, ma sempre trainato dal vento del nuovo che avanza, acceso dal desiderio di miglioramento continuo.

Non sfugge certo a questa dinamica il mondo in cui cammina una rivista scientifica, che per sua natura è alla ricerca del cambiamento e attraverso l'apertura di nuove strade sulle quali contribuire all'approfondimento della conoscenza dei fenomeni oggetto di studio.

Sappiamo bene, però, che cambiare non è cosa facile, nonostante il turbinio del contesto in cui si vive. Non è facile per l'agire di tanti freni al cambiamento tra i quali l'abitudine con il fascino discreto dello status quo che porta con sé, il conformismo, la pressione dell'opinione comune, l'incognita del nuovo, l'affetto che lega le persone ad un contesto particolare.

E questa condizione tende a protrarsi sino a quando un "incidente", un problema, un fatto, una conversazione, uno scontro, la competizione, una "spinta gentile" o quant'altro, non dischiudono le porte a prospettive sino ad allora collocate ai margini dell'orizzonte delle possibili scelte. E contemporaneamente si realizza che l'amore per ciò che si ritiene di lasciare non è legato alle cose, alle forme, bensì alla sostanza e al pensiero che non variano al variare di un agire diverso.

È questo il momento in cui i territori dell'ansia e del timore gradualmente si ritirano.

Così la consapevolezza che se si desidera migliorare non si può che cambiare arriva a dominare le decisioni. Ci si apre ai rischi del nuovo, con lo spirito di chi guarda ad esso con la fiducia e la speranza attiva di poter fare sempre meglio, piuttosto che con l'ansia per ciò che si lascia.

In una cornice di questo tipo, nell'ambito della comunità di Sinergie - quella dei suoi Lettori, degli Autori, del Comitato Scientifico, dei Referee, della Redazione e della Direzione - è maturata la decisione di intensificare il processo di internazionalizzazione della rivista, processo, peraltro, già da tempo avviato con la partecipazione di colleghi stranieri al comitato scientifico e la crescente pubblicazione di articoli in lingua inglese di autori sia italiani che stranieri.

Decisione sostenuta dal desiderio di poter sempre più contribuire alla diffusione del pensiero manageriale italiano al di là dei confini nazionali, creando uno spazio capace di attrarre un dibattito internazionale efficace su temi relativi al divenire dell'impresa e del suo rapportarsi alle istanze del mondo cui partecipa.

Proposito, questo, che ci ha condotto ad interrogarci prima di tutto sull'efficacia del titolo della nostra rivista: Sinergie Rivista di Studi e Ricerche. Titolo che a più riprese vari colleghi avevano proposto di modificare per renderne più chiaro 
l'oggetto di studio, pur senza avanzare allora l'idea di una sua rivisitazione linguistica.

Titolo chela Direzione non si è mai sentita di modificare per la forza evocativa che il termine Sinergie possiede, con il sobrio ed essenziale riferimento alla necessaria apertura interdisciplinare della riflessione sull'impresa per la naturale poliedricità di prospettive nelle quali si rivela.

Tuttavia, oggi è evidente come la carenza nella testata di un riferimento specifico all'oggetto della rivista escluda la possibilità di entrare nei circuiti del dibattito internazionale.

Così, accogliendo il suggerimento in tal senso del Comitato Scientifico, che ha raccomandato di preservare nel cambiamento il marchio Sinergie, si è arrivati alla decisione di mutare quello che era il sottotitolo della rivista trasformando la testata da Sinergie Rivista di Studi e Ricerche in Sinergie Italian Journal of Management.

Abbiamo in questo modo ritenuto di poter idealmente fondere l'italianità della testata, Sinergie rimane espresso in italiano, con il suo desiderio di relazionarsi con il mondo attraverso la propria specifica identità espressa dall'opzione inglese della seconda parte del titolo: Italian Journal of Management.

Ma ovviamente non basta il cambiamento del titolo per realizzare un'apertura internazionale di una rivista.

Così, con la gradualità del caso, e con un'ulteriore apertura internazionale del Comitato Scientifico, Sinergie si propone di passare integralmente alla lingua inglese, riconoscendone il valore di codice linguistico universale della ricerca scientifica, nella prospettiva di accogliere nelle sue pagine sempre più numerosi studiosi di altri Paesi con i quali costruire reti relazionali di ricerca e scambi di conoscenze.

In questo processo anche l'editing della rivista, come pure il suo portale, cambieranno nella direzione di una sua più agevole lettura e della capacità di ospitare tutte le innovazioni dirette a fare di Sinergie Italian Journal of Management un sempre più utile strumento di lavoro per gli studiosi di management $\mathrm{e}$ in particolare per i giovani che decidono di vivere questa avventura.

Allo stesso modo saranno rivisitate le altre due linee editoriali della rivista Sinergie Quaderni e Sinergie Rapporti di Ricerca - per farne agili strumenti dedicati alla diffusione delle ricerche di base condotte dal Cueim e al dialogo con tutte le comunità professionali che incrociano la vita dell'impresa.

Siamo consapevoli della radicalità di questo cambiamento, secondo solo al turnaround della rivista del 1989 vissuto nel momento della morte del suo Fondatore, quando mutarono editing e struttura e si adottò una configurazione monografica pur aperta anche ad una varietà di contributi.

Ciò nondimeno, affrontiamo con entusiasmo e fervore questa sfida forti dello spirito di servizio per la nostra comunità che ha animato la rivista sin dalla sua nascita.

Così, augurandoci di aver ben interpretato i suggerimenti che ci sono pervenuti e contando su un comune lavoro, ribadiamo il nostro impegno a fare il massimo perché Sinergie possa proporsi sempre più come strumento capace di contribuire alla 
valorizzazione della ricerca scientifica nell'ambito delle discipline aziendali, attraverso la costruzione di ponti tra accademia e impresa che possano contribuire alla crescita ed alla diffusione del benessere e della qualità della vita in un contesto di sostenibilità e armonia tra produzione e società.

Claudio Baccarani

Gaetano M. Golinelli 
